\title{
Extended lymphadenectomy in locally advanced rectal cancers: a systematic review
}

\author{
Balaji Mahendran, Supriya Balasubramanya, Simone Sebastiani, Sebastian Smolarek \\ Department of Colorectal Surgery, University Hospitals Plymouth NHS Trust, Plymouth, United Kingdom
}

Purpose: The surgical treatment of advanced low rectal cancer remains controversial. Extended lymphadenectomy (EL) is the preferred option in the East, especially in Japan, while neoadjuvant radiotherapy is the treatment of choice in the West. This review was undertaken to review available evidence supporting each of the therapies.

Methods: All studies looking at EL were included in this review. A comprehensive search was conducted as per PRISMA guidelines. Primary outcome was defined as 5-year overall survival, with secondary outcomes including 3-year overall survival, 3- and 5-year disease-free survival, length of operation, and number of complications.

Results: Thirty-one studies met the inclusion criteria. There was no significant publication bias. There was statistically significant difference in 5-year survival for patient who underwent EL (odds ratio, 1.34; 95 confidence interval, 0.09-0.5; $\mathrm{P}=0.006)$. There were no differences noted in secondary outcomes except for length of the operations.

Conclusion: There is evidence supporting EL in rectal cancer; however, it is difficult to interpret and not easily transferable to a Western population. Further research is necessary on this important topic.

Keywords: Rectal neoplasms; Total mesorectal excision; Lateral pelvic lymph node excision; Extended lymphadenectomy; Overall survival; Systematic review

\section{INTRODUCTION}

Colorectal cancer (CRC) is one of the commonly diagnosed cancers, being the second leading cancer in women, and the third most common cancer in men [1]. The predicted incidence of CRC might increase to 2.5 million new cases in 2035 [2]. The treatment of advanced cases can be quite complex, especially in the context of rectal cancer. The total mesorectal excision (TME) has been well established in treatment of rectal cancer [3]; however, there is a significant discrepancy in the treatment options of-

Received: Aug 3, 2021 - Accepted: Sep 14, 2021

Correspondence to: Supriya Balasubramanya, MBBS, MS, MRCS

Department of Colorectal Surgery, University Hospitals Plymouth NHS Trust, Derriford Road, Plymouth, Devon PL6 8DH, United Kingdom

Tel: +447444150043, E-mail: s.balasubramanya@nhs.net

ORCID: https://orcid.org/0000-0002-7549-8048

\section{- This systematic review was registered on PROSPERO under identification number CRD42019129940.}

(C) 2022 The Korean Society of Coloproctology

This is an open-access article distributed under the terms of the Creative Commons Attribution NonCommercial License (https://creativecommons.org/licenses/by-nc/4.0) which permits unrestricted noncommercial use, distribution, and reproduction in any medium, provided the original work is properly cited. fered to patients who have locally extensive disease. This would be defined as clinical stage II or III cancer, located at or below the peritoneal reflection, that has developed metastasis to lateral pelvic lymph nodes, i.e., common iliac, internal iliac, external iliac, and obturator nodes. These nodes can be involved in cases of rectal cancer in $21.9 \%$ to $61.1 \%$ of cases [4]. The presence of lateral pelvic lymph node involvement confers poorer prognosis to the patient, even when thought to be adequately treated [5]. As such, treating such cases optimally becomes paramount. However, there is currently a difference in consensus regarding the treatment of these cases, largely divided along geographical lines. Western countries, including the United Kingdom and the United States, do not routinely recommend dissection of the lateral pelvic lymph nodes, instead of focusing on neoadjuvant chemoradiotherapy (CRT) and adequate TME [6, 7]. Eastern countries, particularly Japan, have focused on the lateral pelvic lymph node dissection, extended lymphadenectomy (EL) as a standard treatment for stage II and III low rectal cancer [8]. This review aims to collect all available evidence regarding EL, to assess its feasibility and effectiveness compare its outcomes against standard practice in Western countries. 


\section{METHODS}

\section{Search strategy}

A comprehensive systematic search of the literature was performed in keeping with PRISMA (Preferred Reporting Items for Systematic Reviews and Meta-Analyses) guidelines. Records on PubMed, MEDLINE, Embase, Ovid, Cochrane Library, and Google Scholar were searched for all relevant articles published from January 2008 to January 2019 to give comprehensive current practice review. All articles were published in English language.

The search was performed using the following search terms: "rectal cancer," "extended lymphadenectomy," "lateral pelvic lymph node dissection," "mesorectal excision," "mesorectum," and "lateral lymph node." Abstract and conference proceedings were excluded during preliminary screening due to high risk of incomplete data. Two independent reviewers screened all titles, abstracts, and full-text articles with any disagreement being settled via discussion with the senior author. Publications reporting on the same series of patients were identified and only the most recent data were included.

\section{Types of articles reviewed}

Randomized controlled trials (RCTs), prospective and retrospective observational studies with controls, and retrospective matchedpair studies were all included.

Studies that did not include a specific section on data for patients, and studies that had fewer than 7 patients (defined as case studies [9]), non-English publications, or studies reported on pelvic exenterations were excluded.

\section{Types of participants}

The studies included cases of adults diagnosed with rectal cancer treated by operation with curative intent, which was either standard TME or TME with pelvic sidewall EL.

\section{Types of intervention and comparators}

Only studies looking at the operative treatment for locally advanced rectal cancer with or without involved pelvic side lymph nodes, with or without preoperative radiotherapy were included. Comparators were TME vs. EL.

Primary outcome was defined as 5-year overall survival (OS) after TME or EL. Secondary outcomes included 3-year OS, 3- and 5-year disease-free survival (DFS), local and distal recurrence rate, length of operation, number of complications, and volume of blood loss.

\section{Data extraction}

Two independent reviewers extracted the data from the papers using a specially designed extraction form. The following data were collected (if reported): 5- and 3-year survival, DFS, OS, number of distant and local recurrence, length of operations, blood loss, and number of postoperative complications.

\section{Data analysis}

The methodological quality of the study was assessed using the revised Cochrane risk of bias tool to assess in randomized trials [10]. Nonrandomized studies were assessed using the methodological index for nonrandomized studies (MINORS) score [11]. Noncomparative studies scored a maximum of 16 and 24 for comparative papers. Two authors scored all articles included for review independently.

Publication bias was checked by plotting the papers on the Funnel plot and using the Egger test with random effect. The heterogeneity of the study was checked according to Cochrane Collaboration's Handbook using Q parameter and $\mathrm{I}^{2}$ statistics. $\mathrm{I}^{2}$ less than $40 \%$ was considered low heterogeneity. Forrest plot and odds ratio (OR) with $95 \%$ confidence interval (CI) were used to compare studies and display the results. The P-value for overall effect was calculated using Z-test. All statistical analyses were made with $\mathrm{R}$ language with metafor package (R Foundation for Statistical Computing, Vienna, Austria). Difference between medians was assessed using quantile estimation according to McGrath et al. [12].

\section{RESULTS}

\section{Search results}

The study selection process is outlined in the PRISMA diagram (Fig. 1). The initial search identified 414 papers. After removing duplications, 307 studies were included in the further text and abstract review. Further 229 studies were excluded after abstract review as they were not relevant, including conference proceedings and abstracts. Seventy-eight papers were reviewed in full text including all references to help identify any other relevant articles not identified through initial search. A further 46 were excluded for not meeting the inclusion criteria (articles not being relevant, 35; case studies, 1, articles with subgroup analysis from 1 RCT, 9; insufficient data available, 1). Finally, 31 articles were included for the systematic review [13-43]. There were no additional studies identified through other sources. The methodological qualities of the studies are represented in Table 1 and Fig. 2. The mean MINORS score was 10.5 with a standard deviation of 3.7 pointing toward low-quality studies (Table 1).

\section{Publication bias and heterogeneity}

Calculation of the publication bias was done for primary outcome studies. The Funnel plot was symmetrical and Egger test for mixedeffect meta-regression model was not statistically significant with $\mathrm{Z}=-0.12, \mathrm{P}=0.91$ pointing toward the lack of bias (Fig. 3).

\section{Study characteristics}

A total of 5,240 patients underwent an EL for the resection of rectal cancer. The major characteristics of these patients are shown in Table 2. Some papers did not publish the mean age for the 2 groups of patients being studied, or published data in a nonstan- 


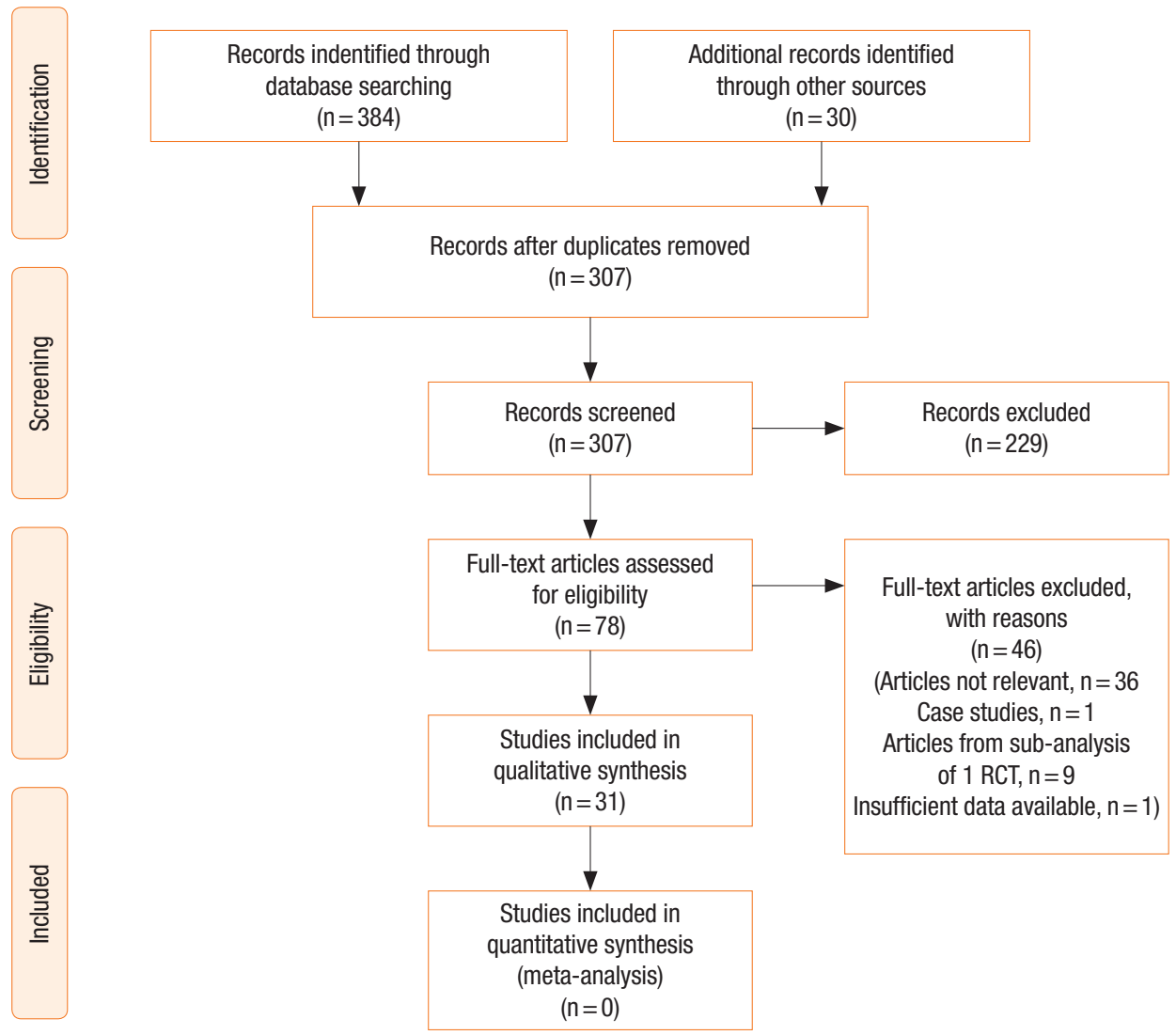

Fig. 1. Study selection process. RCT, randomized controlled trial.

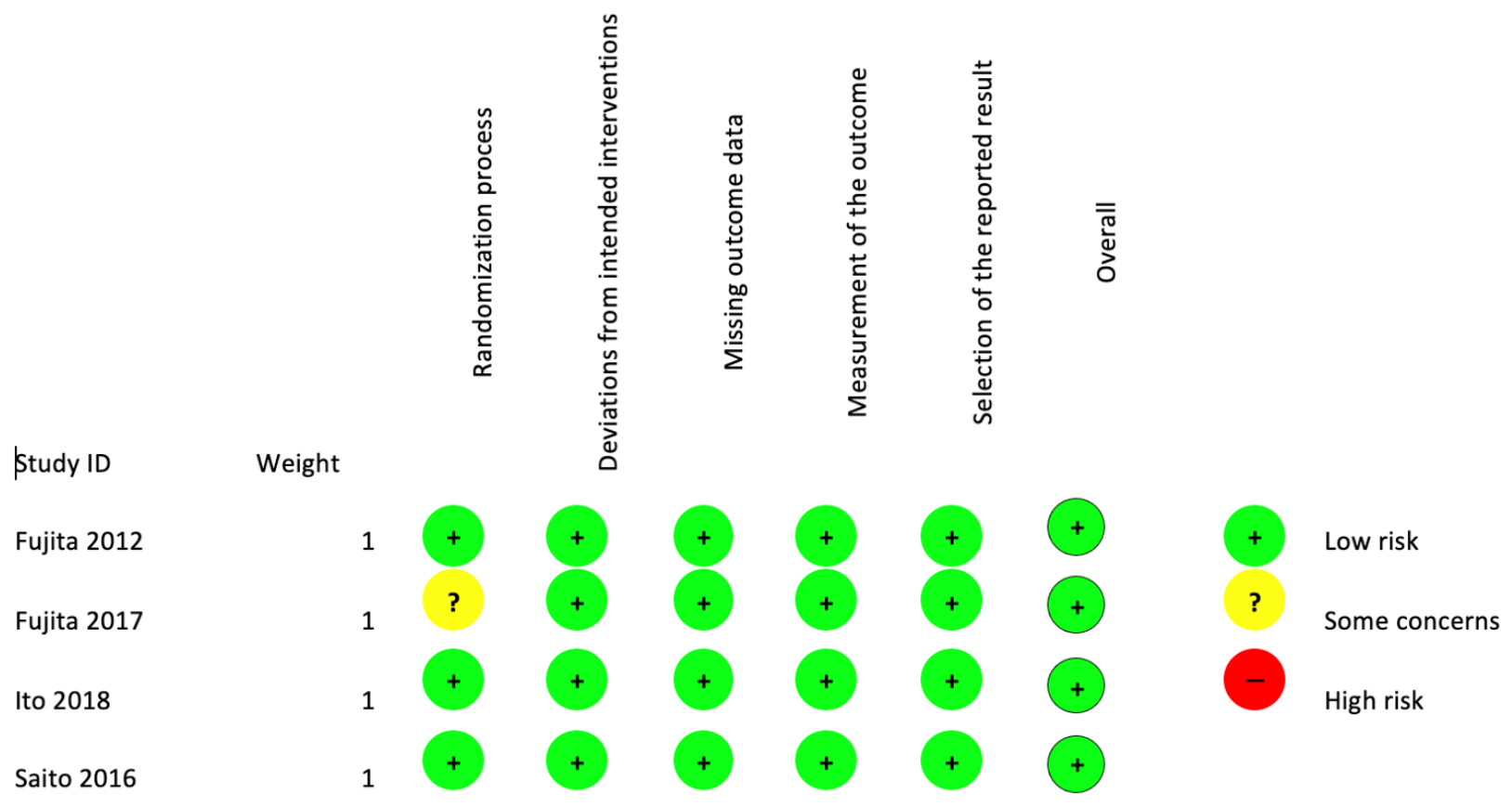

Fig. 2. Cochrane risk of bias assessment of randomized controlled trials. 


\section{Coloproctology Balaji Mahendran, et al.}

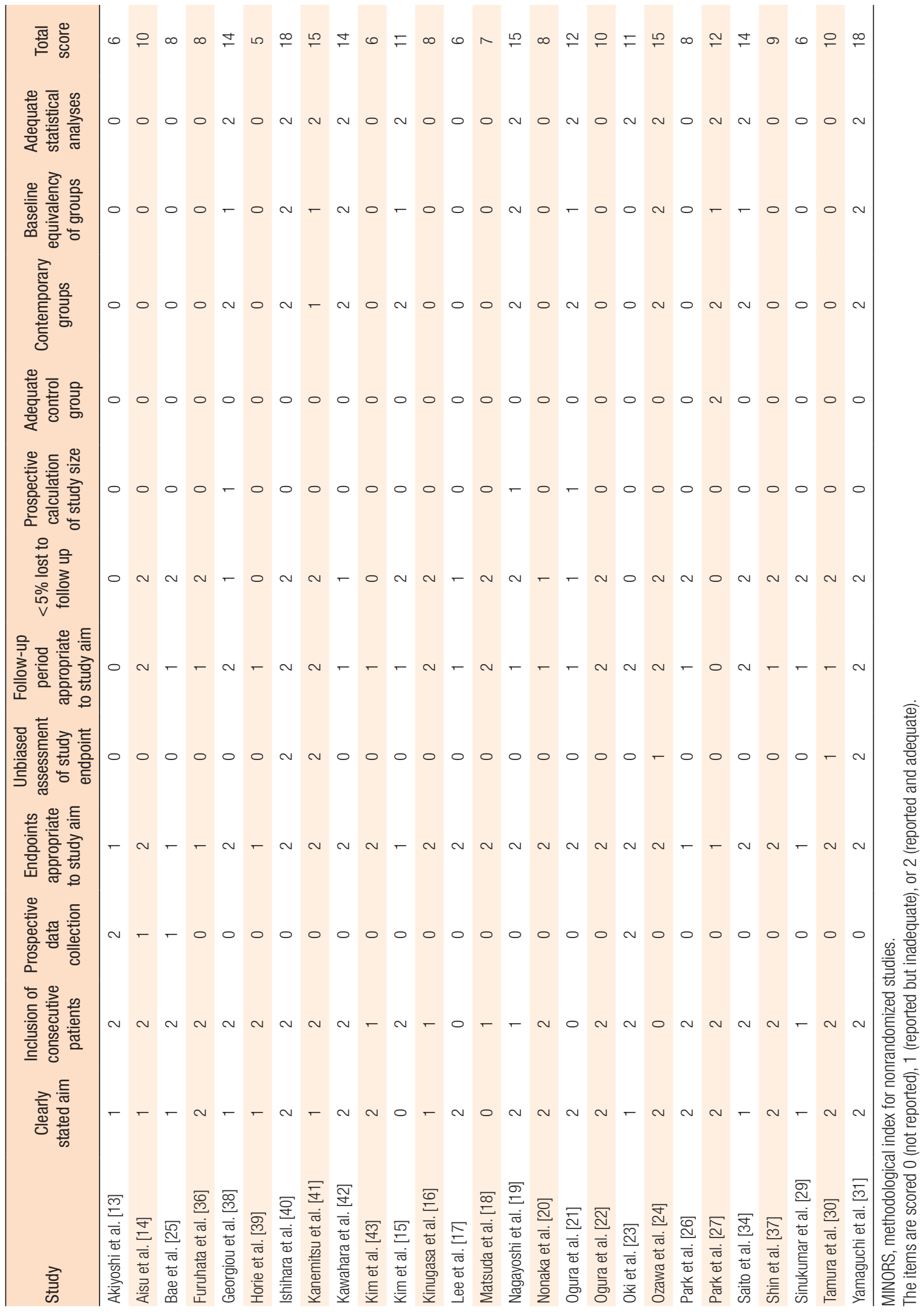




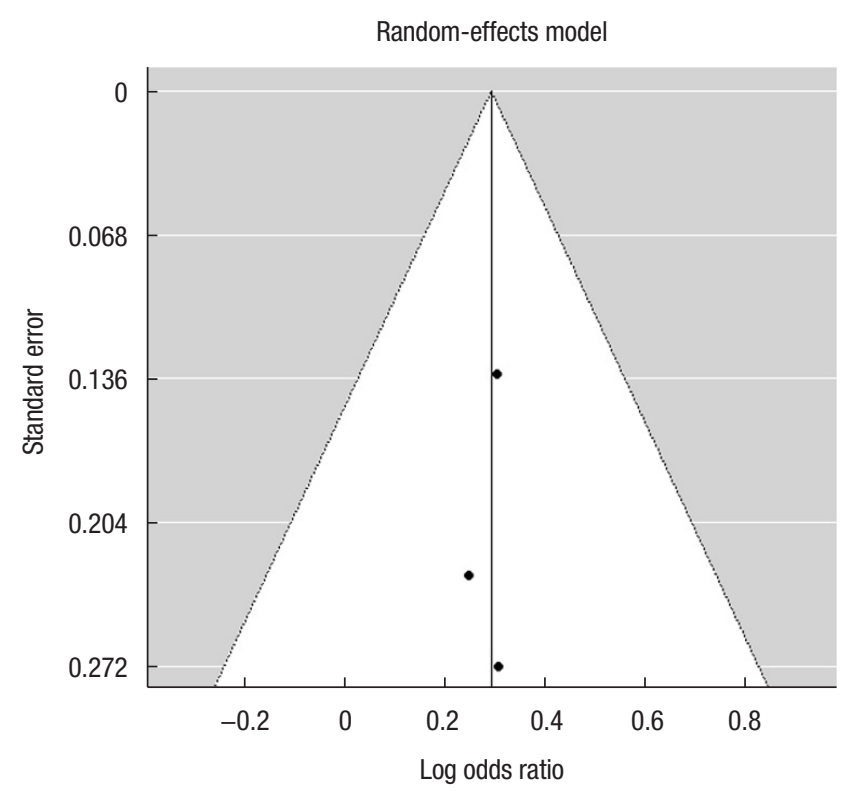

Fig. 3. Funnel plot for calculation of publication bias.

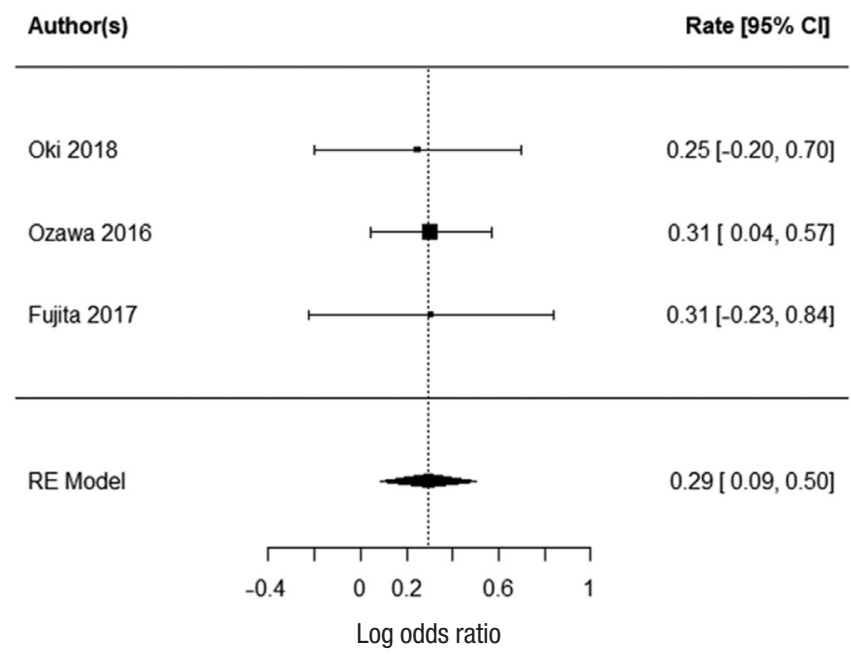

Fig. 4. Forrest plot for primary outcome (5-year overall survival). CI, confidence interval. RE, random-effects.

dard way $[13,16,20,24,25,29,30,36,43]$, and were not included in the calculation of median age for these patients. The same difficulty arose with the gender breakdown of the patients included in these studies. Furthermore, some papers compared different techniques in performing EL $[15,31]$ or EL after neoadjuvant chemotherapy [14]. Where possible this data has been included into the overall EL cohort for analysis.

\section{Primary outcome}

Three studies report 5-year survival; Oki et al. [23], Ozawa et al. [24], and Fujita et al. [28]. There was no heterogeneity with the
Author(s)

Rate $[95 \% \mathrm{Cl}]$

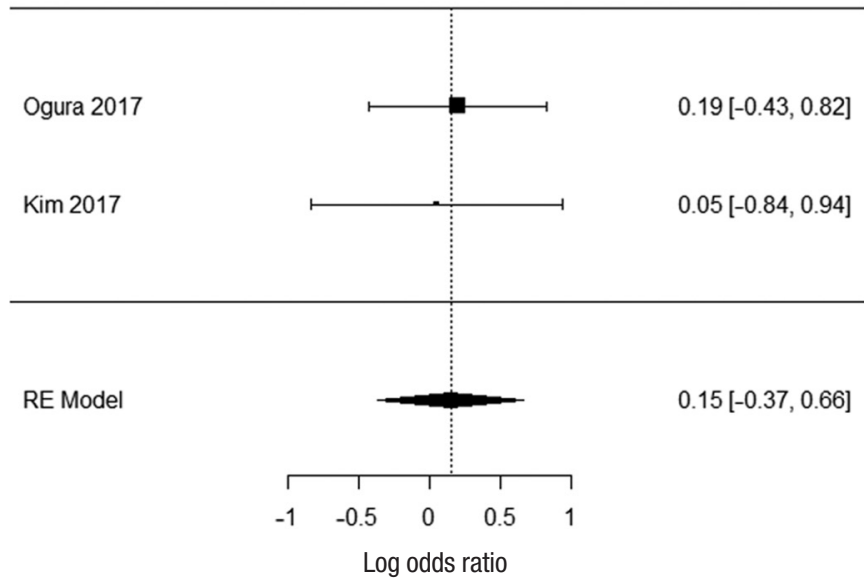

Fig. 5. Forrest plot for 3-year overall survival. CI, confidence interval. RE, random-effects.

data $\left(\mathrm{I}^{2}=0 \%\right)$. There was a statistically significant difference in 5 -year survival for patients who underwent EL. The summarized random effect is $\mathrm{P}=0.006$ (OR, 1.34; $95 \mathrm{CI}, 0.09-0.5)$ (Fig. 4).

\section{Secondary outcomes}

Three-year overall survival

Two studies report 3-year OS; Ogura et al. [21] and Kim et al. [43]. There was no heterogeneity between the study data $\left(\mathrm{I}^{2}=0 \%\right)$. There was no statistically significant difference in 3-year survival for patients who underwent EL. The summarized random effect is $\mathrm{P}=0.55$ (OR, 1.27; $95 \mathrm{CI},-0.55-1.03$ ) (Fig. 5).

\section{Three-year and 5-year disease-free survival}

Two studies reported 3-year DFS; Ogura et al. [21] and Kim et al. [43]. There was no heterogeneity between the study data $\left(I^{2}=0 \%\right)$. There was no statistically significant difference in 3-year DFS for patients who underwent EL. The summarized random effect is $\mathrm{P}=0.57$ (OR, 1.16; 95 CI, -0.37-0.66) (Fig. 6). Five-year DFS was reported in 2 studies; Oki et al. [23] and Fujita et al. [28]. There was weak heterogeneity between the study $\mathrm{I}^{2}=27.34 \%$. There was no statistically significant difference in 5-year DFS for patients who underwent EL. The summarized random effect is $\mathrm{P}=0.15$ (OR, 1.27; 95 CI, -0.09-0.57) (Fig. 7).

\section{Local recurrence}

Six paper reported the number of local and distal recurrence $[15$, $17,23,28,40,43]$. For local recurrence, there was significant heterogeneity between the studies $\left(I^{2}=79.2 \%\right)$; therefore, it was not possible to summarize it with random effect. The reported mean local recurrence rate was $10.2 \%$ (range, 6\%-24.3\%) for EL and $12 \%$ (range, $0 \%-22.9 \%$ ) for TME. The 3 papers that reported distal recurrence $[15,17,23]$ at $66 \%, 10 \%$, and $13 \%$, respectively had low heterogeneity between them $\left(\mathrm{I}^{2}=0 \%\right)$. The summarized ran- 


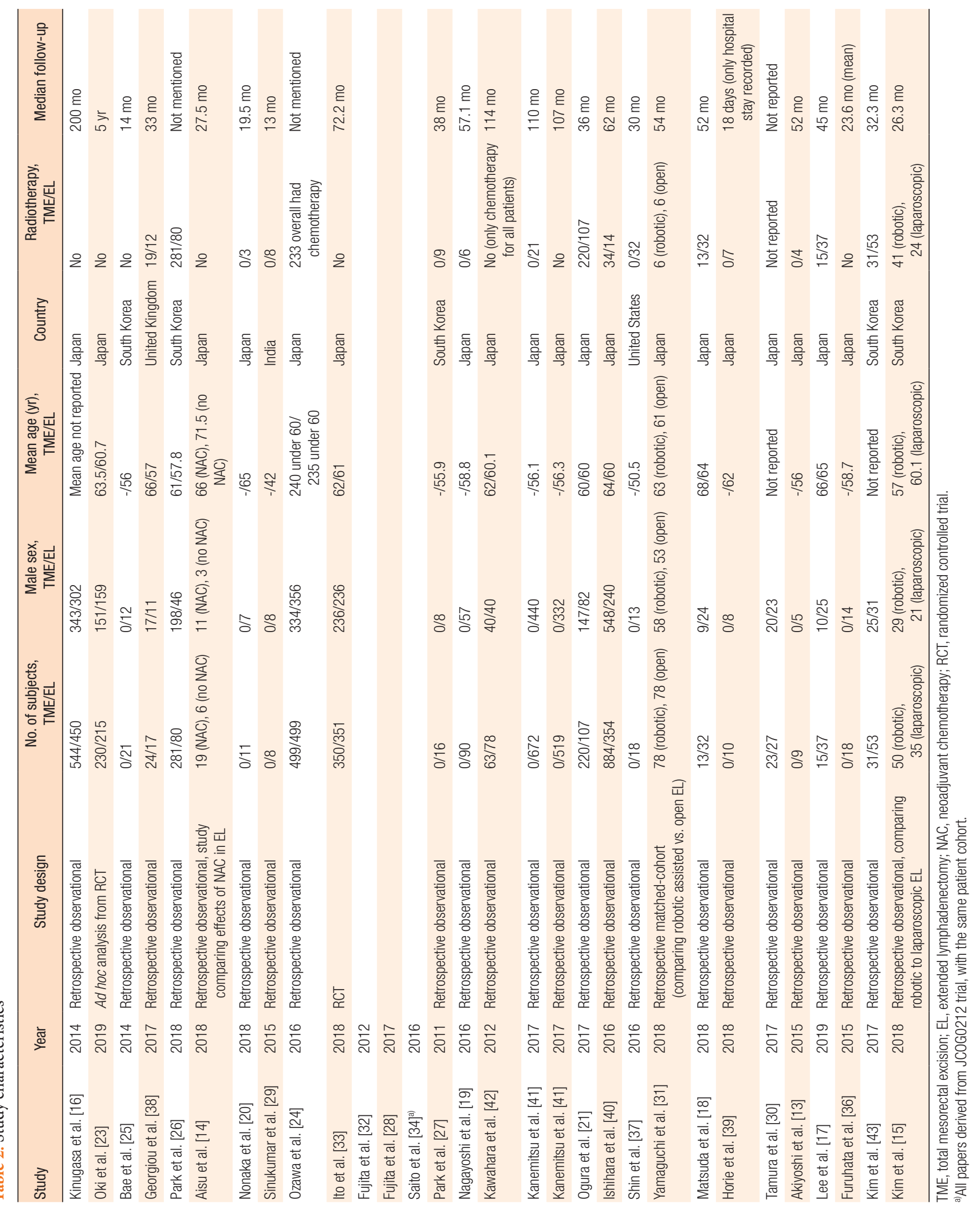




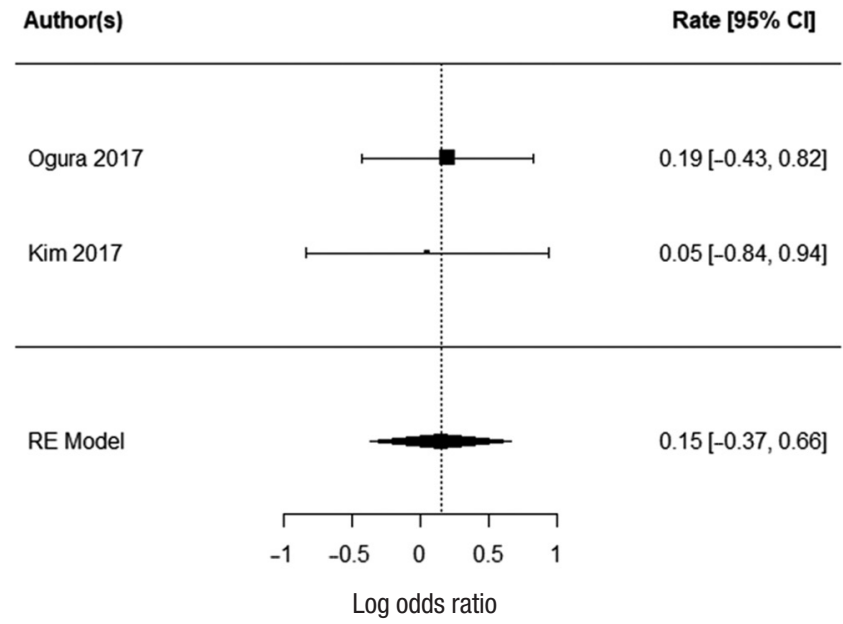

Fig. 6. Forrest plot for 3-year disease-free survival. CI, confidence interval. RE, random-effects.

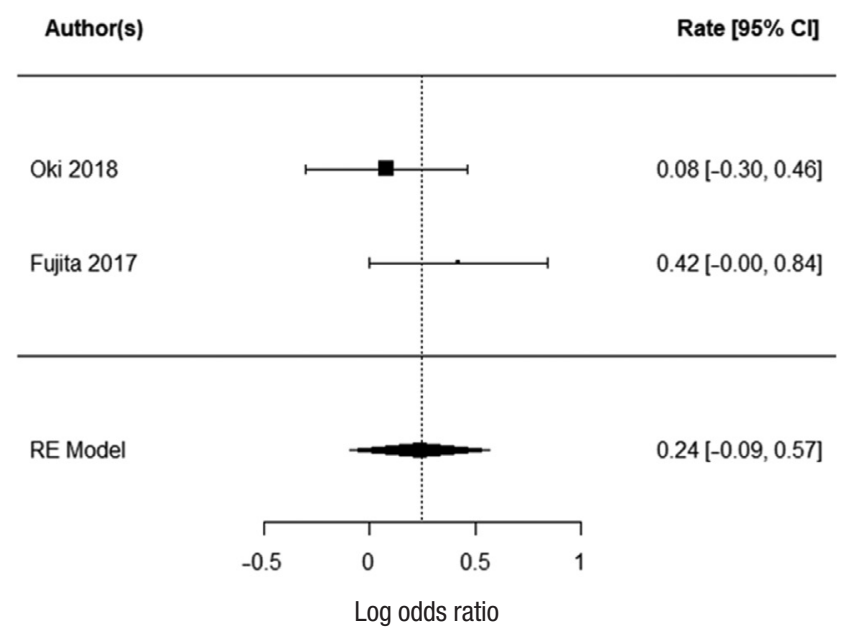

Fig. 7. Forrest plot for 5-year disease-free survival. CI, confidence interval. RE, random-effects.

dom effect was not statistically significant $(\mathrm{P}=0.25$; $\mathrm{OR}, 1.25$; 95 CI, -0.15-0.59) (Fig. 8).

\section{Other surgical outcomes}

Five papers reported the length of operation [17, 18, 21, 26, 33]. There was low heterogeneity between the studies $\left(\mathrm{I}^{2}=0 \%\right)$. The EL was significantly longer than TME with summarized random effect $(\mathrm{P}<0.0001 ; 95 \mathrm{CI}, 94.03-122.10)$. Three papers reported the numbers of complications $[17,18,21]$ with low heterogeneity $\left(\mathrm{I}^{2}=0 \%\right)$. The summarized random effect was not statistically significant ( $\mathrm{P}=0.17$; OR, 1.36; 95 CI, 0.013-0.75) (Fig. 9).

Five papers report the blood loss after TME and EL [17, 18, 21, $26,33]$ (Table 3). There was significant heterogeneity between the papers with $\mathrm{I}^{2}=87.41 \%$ therefore it was not possible to summa-

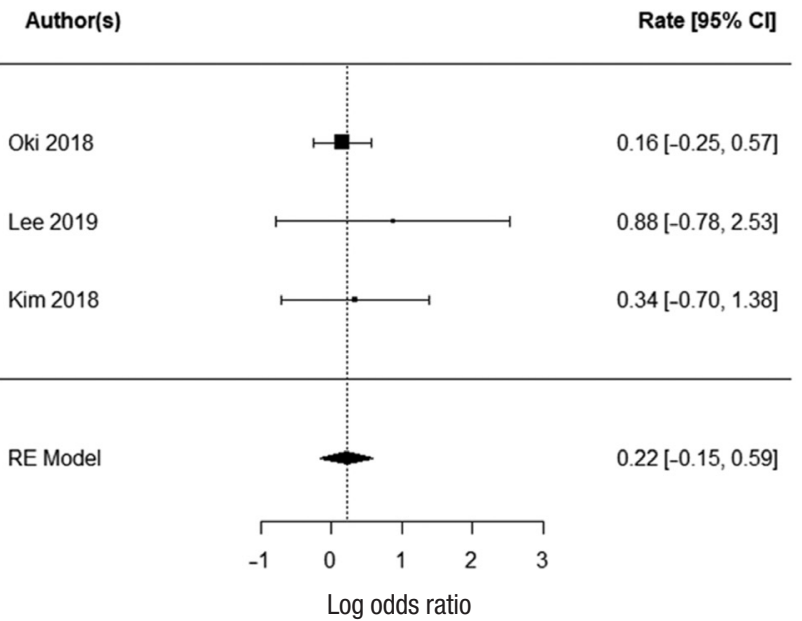

Fig. 8. Forrest plot for distal recurrence. CI, confidence interval. RE, random-effects.

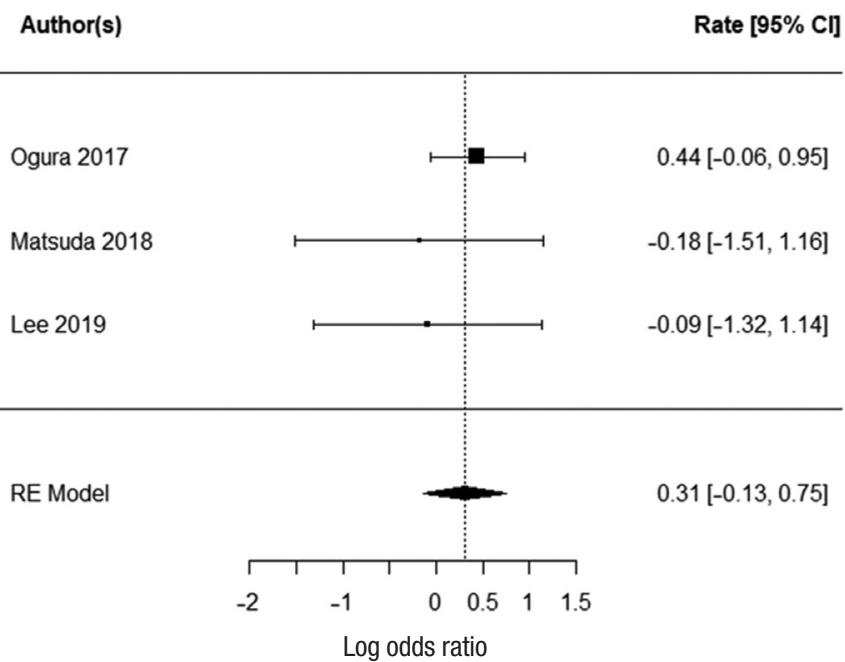

Fig. 9. Forrest plot for complications. CI, confidence interval. RE, random-effects.

rize it with random effect. The reported mean blood loss for EL was $560 \mathrm{~mL}$ (range, 100-582 mL) and $135 \mathrm{~mL}$ (range, 30-337 $\mathrm{mL}$ ) for TME.

\section{DISCUSSION}

There is evidence in support EL in rectal cancer patients, improving their 5-year OS. However, there is some difficulty in interpreting these results, as this benefit is not seen in any of the 3-year OS, 3-year DFS, 5-year DFS, or distal recurrence. Only 3 papers reported their 5-year OS. Their individual rates of 5-year OS are presented in Table 4, reporting rates between $79.9 \%$ and $92.6 \%$. It is also important to note that Fujita et al. [28] was a large multi- 


\section{Coloproctology $\quad$ Balaji Mahendran, et al.}

Table 3. Average blood loss as reported in studies

\begin{tabular}{lccr}
\hline \multirow{2}{*}{ Study } & \multirow{2}{*}{ Year } & \multicolumn{2}{c}{ Average blood loss (mL) } \\
\cline { 3 - 4 } & & EL & TME \\
\hline Ogura et al. [21] & 2017 & 115 & 30 \\
Matsuda et al. [18] & 2018 & 582 & 300 \\
Lee et al. [17] & 2019 & 560 & 135 \\
Park et al. [26] & 2018 & 100 & 100 \\
Ito et al. [33] & 2018 & 576 & 337 \\
\hline
\end{tabular}

EL, extended lymphadenectomy; TME, total mesorectal excision.

center RCT (JCOG0212). This high-quality paper concluded that the noninferiority of TME alone was not demonstrated, with regards to the primary outcome of a 5-year DFS. It did however use a 90.9\% CI to assess the upper limit of the range of the hazard ratio, rather than the accepted $95 \%$. Indeed there were no significant differences in the 5-year OS and 5-year local recurrence-free survival. There was, however, a significantly lower rate of local recurrence in the EL group when compared to the TME group. This is in keeping with what is currently thought to be the justification for an EL. The other difficulty with assessing the data from JCOG0212 is that EL was performed as a prophylactic measure and none of the patients received radio or CRT to the pelvis which is currently not recommended as a standard practice in stage III cancer in Japan. This is contrary to standard practice in Western countries where radiotherapy to the pelvis is frequently used to downgrade the tumor. Moreover, patients were only included in JCGO212 if they had no extramesorectal lateral lymph node enlargement on preoperative magnetic resonance imaging. This is also contrary to the Western practice where EL is recommended to the cases with enlarged pelvic sidewall nodes, especially persistent after radiotherapy.

Local recurrence is an important factor in considering the merits of EL over a TME. We were not able to summarize the risk of local recurrence in our review; however, there was no difference in the rates of distal recurrence. This brings into question the homogeneity of these patients, and the views of the surgical community with regards to the labeling of the spread of this disease. Western countries consider pelvic lymphadenopathy a sign of systemic disease, whereas the Japanese cohort considers this a local spread of cancer [44]. The effects of local recurrence are also uncertain, with its impact on survival and functional outcomes before and after CRT not well studied.

As expected, EL took longer to perform than TME. This is not in itself a poor prognosticator; however, using the number of all complications as a secondary marker would suggest that it does not affect the patient on average. These procedures were mostly performed by high-volume centers, suggesting a limitation for how short the procedure can be even when performed by experienced surgeons. Additionally, one needs to take into consideration that EL in Western practice is significantly less common;
Table 4. Five-year overall survival in studies comparing EL to TME resections

\begin{tabular}{lcccccc}
\hline \multirow{2}{*}{ Study } & \multirow{2}{*}{ Year } & \multicolumn{2}{c}{ Sample size (n) } & & \multicolumn{2}{c}{ Five-year survival (\%) } \\
\cline { 3 - 4 } \cline { 7 - 7 } & & EL & TME & & EL & TME \\
\hline Oki et al. [23] & 2018 & 215 & 230 & & 79.9 & 75.6 \\
Ozawa et al. [24] & 2016 & 499 & 499 & & 68.9 & 62.0 \\
Fujita et al. [28] & 2017 & 351 & 350 & & 92.6 & 90.2 \\
\hline
\end{tabular}

EL, extended lymphadenectomy; TME, total mesorectal excision.

therefore, the operative time and number of postoperative complications may be higher than reported.

It is difficult to have an overall impression regarding the efficacy of EL over TME and radiotherapy. The biology of these tumors is difficult to study, with regards to them being aggressive either locally or distally. Our review suggests there is a partial benefit to performing EL in these patients. However, it is important to note that in performing an EL, many at-risk structures are encountered, including pelvis blood vessels and nerves. These confer a significant effect on the quality of life of such patients. The American National Comprehensive Cancer Network guidelines suggest an EL is not indicated in the absence of clinically suspected nodes, and some studies have suggested that the effect of neoadjuvant CRT is enough to reduce the number of clinically evident lymph nodes in a rectal resection specimen [45]. There is clearly a need to consider standardization of therapy, as even within the United Kingdom there are varying numbers of therapies used to treat such patients [46]. The JCOG0212 study is a step in the right direction in assessing the efficacy of such treatment, showing promising results. There will be difficulty with regards to the applicability of such a study in the Western population, and this is good point to consider a multicenter RCT or well-designed observational study in the Western population of patients with stage III rectal cancer and positive nodes in the pelvic sidewall.

There is evidence supporting EL in stage III rectal cancer; however, there are difficulties in the applicability of these results straight to clinical practice in the Western population. This is caused by the lack of homogeneity of the populations being studied, especially in relation to the use of preoperative pelvic radiotherapy. Further studies focusing on the Western population would be an important next step in evaluating this treatment modality.

\section{CONFLICT OF INTEREST}

No potential conflict of interest relevant to this article was reported.

\section{REFERENCES}

1. Dekker E, Tanis PJ, Vleugels JLA, Kasi PM, Wallace MB. Colorec- 
tal cancer. Lancet 2019;394:1467-80.

2. Bray F, Ferlay J, Soerjomataram I, Siegel RL, Torre LA, Jemal A. Global cancer statistics 2018: GLOBOCAN estimates of incidence and mortality worldwide for 36 cancers in 185 countries. CA Cancer J Clin 2018;68:394-424.

3. Heald RJ, Ryall RD. Recurrence and survival after total mesorectal excision for rectal cancer. Lancet 1986;1:1479-82.

4. Atef Y, Koedam TW, van Oostendorp SE, Bonjer HJ, Wijsmuller AR, Tuynman JB. Lateral pelvic lymph node metastases in rectal cancer: a systematic review. World J Surg 2019;43:3198-206.

5. Kim TH, Jeong SY, Choi DH, Kim DY, Jung KH, Moon SH, et al. Lateral lymph node metastasis is a major cause of locoregional recurrence in rectal cancer treated with preoperative chemoradiotherapy and curative resection. Ann Surg Oncol 2008;15:72937.

6. National Comprehensive Cancer Network (NCCN). NCCN Clinical Practice Guidelines in Oncology: Rectal Cancer. Version 1. Plymouth Meeting (PA): NCCN Foundation; 2020.

7. National Comprehensive Cancer Network (NCCN). NCCN Clinical Practice Guidelines in Oncology: Colorectal Cancer. Version 1. Plymouth Meeting (PA): NCCN Foundation; 2020.

8. Konishi T. Management of lateral pelvic lymph node in the East: time to learn from the Western viewpoints. Ann Gastroenterol Surg 2018;2:330-1.

9. Moher D, Liberati A, Tetzlaff J, Altman DG; PRISMA Group. Preferred reporting items for systematic reviews and meta-analyses: the PRISMA statement. BMJ 2009;339:b2535.

10. Sterne JAC, Savović J, Page MJ, Elbers RG, Blencowe NS, Boutron I, et al. RoB 2: a revised tool for assessing risk of bias in randomised trials. BMJ 2019;366:14898.

11. Slim K, Nini E, Forestier D, Kwiatkowski F, Panis Y, Chipponi J. Methodological index for non-randomized studies (MINORS): development and validation of a new instrument. ANZ J Surg 2003;73:712-6.

12. McGrath S, Sohn H, Steele R, Benedetti A. Meta-analysis of the difference of medians. Biom J 2020;62:69-98.

13. Akiyoshi T, Nagata J, Nagasaki T, Konishi T, Fujimoto Y, Nagayama S, et al. Laparoscopic salvage lateral pelvic lymph node dissection for locally recurrent rectal cancer. Colorectal Dis 2015;17: O213-6.

14. Aisu Y, Kato S, Kadokawa Y, Yasukawa D, Kimura Y, Takamatsu Y, et al. Feasibility of extended dissection of lateral pelvic lymph nodes during laparoscopic total mesorectal excision in patients with locally advanced lower rectal cancer: a single-center pilot study after neoadjuvant chemotherapy. Med Sci Monit 2018;24: 3966-77.

15. Kim HJ, Choi GS, Park JS, Park SY, Lee HJ, Woo IT, et al. Selective lateral pelvic lymph node dissection: a comparative study of the robotic versus laparoscopic approach. Surg Endosc 2018;32:246673.

16. Kinugasa T, Akagi Y, Shirouzu K. Benefit of lateral lymph node dissection for rectal cancer: long-term analysis of 944 cases un- dergoing surgery at a single center (1975-2004). Anticancer Res 2014;34:4633-9.

17. Lee D, Matsuda T, Yamashita K, Hasegawa H, Yamamoto M, Kanaji S, et al. Significance of lateral pelvic lymph node size in predicting metastasis and prognosis in rectal cancer. Anticancer Res 2019;39:993-8.

18. Matsuda T, Sumi Y, Yamashita K, Hasegawa H, Yamamoto M, Matsuda Y, et al. Outcomes and prognostic factors of selective lateral pelvic lymph node dissection with preoperative chemoradiotherapy for locally advanced rectal cancer. Int J Colorectal Dis 2018;33:367-74.

19. Nagayoshi K, Ueki T, Manabe T, Moriyama T, Yanai K, Oda Y, et al. Laparoscopic lateral pelvic lymph node dissection is achievable and offers advantages as a minimally invasive surgery over the open approach. Surg Endosc 2016;30:1938-47.

20. Nonaka T, Fukuda A, Maekawa K, Nagayoshi S, Tokunaga T, Takatsuki M, et al. The feasibility and efficacy of laparoscopic extended total mesorectal excision for locally advanced lower rectal cancer. In Vivo 2018;32:643-8.

21. Ogura A, Akiyoshi T, Nagasaki T, Konishi T, Fujimoto Y, Nagayama S, et al. Feasibility of laparoscopic total mesorectal excision with extended lateral pelvic lymph node dissection for advanced lower rectal cancer after preoperative chemoradiotherapy. World J Surg 2017;41:868-75.

22. Ogura A, Konishi T, Cunningham C, Garcia-Aguilar J, Iversen H, Toda S, et al. Neoadjuvant (chemo)radiotherapy with total mesorectal excision only is not sufficient to prevent lateral local recurrence in enlarged nodes: results of the multicenter lateral node study of patients with low cT3/4 rectal cancer. J Clin Oncol 2019; 37:33-43.

23. Oki E, Shimokawa M, Ando K, Murata A, Takahashi T, Maeda K, et al. Effect of lateral lymph node dissection for mid and low rectal cancer: an ad-hoc analysis of the ACTS-RC (JFMC35-C1) randomized clinical trial. Surgery 2019;165:586-92.

24. Ozawa H, Kotake K, Hosaka M, Hirata A, Sugihara K. Impact of lateral pelvic lymph node dissection on the survival of patients with T3 and T4 low rectal cancer. World J Surg 2016;40:1492-9.

25. Bae SU, Saklani AP, Hur H, Min BS, Baik SH, Lee KY, et al. Robotic and laparoscopic pelvic lymph node dissection for rectal cancer: short-term outcomes of 21 consecutive series. Ann Surg Treat Res 2014;86:76-82.

26. Park BK, Lee SJ, Hur BY, Kim MJ, Chan Park S, Chang HJ, et al. Feasibility of selective lateral node dissection based on magnetic resonance imaging in rectal cancer after preoperative chemoradiotherapy. J Surg Res 2018;232:227-33.

27. Park JS, Choi GS, Lim KH, Jang YS, Kim HJ, Park SY, et al. Laparoscopic extended lateral pelvic node dissection following total mesorectal excision for advanced rectal cancer: initial clinical experience. Surg Endosc 2011;25:3322-9.

28. Fujita S, Mizusawa J, Kanemitsu Y, Ito M, Kinugasa Y, Komori K, et al. Mesorectal excision with or without lateral lymph node dissection for clinical stage II/III lower rectal cancer (JCOG0212): a 
multicenter, randomized controlled, noninferiority trial. Ann Surg 2017;266:201-7.

29. Sinukumar S, Engineer R, Saklani A. Preliminary experience with lateral pelvic lymph node dissection in locally advanced rectal cancer. Indian J Gastroenterol 2015;34:320-4.

30. Tamura H, Shimada Y, Kameyama H, Yagi R, Tajima Y, Okamura T, et al. Prophylactic lateral pelvic lymph node dissection in stage IV low rectal cancer. World J Clin Oncol 2017;8:412-9.

31. Yamaguchi T, Kinugasa Y, Shiomi A, Kagawa H, Yamakawa Y, Furutani A, et al. Oncological outcomes of robotic-assisted laparoscopic versus open lateral lymph node dissection for locally advanced low rectal cancer. Surg Endosc 2018;32:4498-505.

32. Fujita S, Akasu T, Mizusawa J, Saito N, Kinugasa Y, Kanemitsu Y, et al. Postoperative morbidity and mortality after mesorectal excision with and without lateral lymph node dissection for clinical stage II or stage III lower rectal cancer (JCOG0212): results from a multicentre, randomised controlled, non-inferiority trial. Lancet Oncol 2012;13:616-21.

33. Ito M, Kobayashi A, Fujita S, Mizusawa J, Kanemitsu Y, Kinugasa Y, et al. Urinary dysfunction after rectal cancer surgery: results from a randomized trial comparing mesorectal excision with and without lateral lymph node dissection for clinical stage II or III lower rectal cancer (Japan Clinical Oncology Group Study, JCOG0212). Eur J Surg Oncol 2018;44:463-8.

34. Saito S, Fujita S, Mizusawa J, Kanemitsu Y, Saito N, Kinugasa Y, et al. Male sexual dysfunction after rectal cancer surgery: Results of a randomized trial comparing mesorectal excision with and without lateral lymph node dissection for patients with lower rectal cancer: Japan Clinical Oncology Group Study JCOG0212. Eur J Surg Oncol 2016;42:1851-8.

35. Ouyang M, Liao T, Lu Y, Deng L, Luo Z, Wu J, et al. Laparoscopic versus open surgery in lateral lymph node dissection for advanced rectal cancer: a meta-analysis. Gastroenterol Res Pract 2019;2019:7689082.

36. Furuhata T, Okita K, Nishidate T, Ito T, Yamaguchi H, Ueki T, et al. Clinical feasibility of laparoscopic lateral pelvic lymph node dissection following total mesorectal excision for advanced rectal cancer. Surg Today 2015;45:310-4.
37. Shin US, Nancy You Y, Nguyen AT, Bednarski BK, Messick C, Maru DM, et al. Oncologic outcomes of extended robotic resection for rectal cancer. Ann Surg Oncol 2016;23:2249-57.

38. Georgiou PA, Mohammed Ali S, Brown G, Rasheed S, Tekkis PP. Extended lymphadenectomy for locally advanced and recurrent rectal cancer. Int J Colorectal Dis 2017;32:333-40.

39. Horie H, Koinuma K, Ito H, Sadatomo A, Naoi D, Kono Y, et al. Utility of preoperative 3-D simulation of laparoscopic lateral pelvic lymph node dissection for advanced rectal cancer: surgical outcomes of 10 initial cases. Asian J Endosc Surg 2018;11:355-61.

40. Ishihara S, Kanemitsu Y, Murono K, Otani K, Yasuda K, Nishikawa $\mathrm{T}$, et al. Oncological benefit of lateral pelvic lymph node dissection for rectal cancer treated without preoperative chemoradiotherapy: a multicenter retrospective study using propensity score analysis. Int J Colorectal Dis 2016;31:1315-21.

41. Kanemitsu Y, Komori K, Shida D, Ochiai H, Tsukamoto S, Kinoshita T, et al. Potential impact of lateral lymph node dissection (LLND) for low rectal cancer on prognoses and local control: a comparison of 2 high-volume centers in Japan that employ different policies concerning LLND. Surgery 2017;162:303-14.

42. Kawahara H, Watanabe K, Ushigome T, Yanagisawa S, Kobayashi $S$, Yanaga K. Lateral pelvic lymph node dissection using laterovesical approach with aspiration procedure for advanced lower rectal cancer. Hepatogastroenterology 2012;59:116-9.

43. Kim HJ, Choi GS, Park JS, Park SY, Cho SH, Lee SJ, et al. Optimal treatment strategies for clinically suspicious lateral pelvic lymph node metastasis in rectal cancer. Oncotarget 2017;8:100724-33.

44. Christou N, Meyer J, Toso C, Ris F, Buchs NC. Lateral lymph node dissection for low rectal cancer: is it necessary? World J Gastroenterol 2019;25:4294-9.

45. Wichmann MW, Müller C, Meyer G, Strauss T, Hornung HM, Lau-Werner $\mathrm{U}$, et al. Effect of preoperative radiochemotherapy on lymph node retrieval after resection of rectal cancer. Arch Surg 2002;137:206-10.

46. Jones HG, Radwan RW, Sams E, Gibby M, Coomer W, Jeffries J, et al. Incidence and treatment of positive pelvic sidewall lymph nodes in patients with rectal cancer. Colorectal Dis 2020;22:1560-7. 